IRSTI 27.35.51

\title{
Charging of a rodlike grain in plasma flow
}

\author{
R.I. Golyatina ${ }^{1}$ and S.A. Maiorov ${ }^{1,2 *}$ \\ ${ }^{1}$ Prokhorov General Physics Institute, Russian Academy of Sciences, \\ 38, Vavilov Str., 119991, Moscow, Russia \\ ${ }^{2}$ Joint Institute for High Temperatures, Russian Academy of Sciences, \\ 13, Izhorskaya Str., bd. 2, 127412, Moscow, Russia \\ e-mail: mayorov_sa@mail.ru
}

\begin{abstract}
In present work on the basis of numerical modeling by the dynamics of many particles, a self-consistent problem of the formation of a charge accumulated by a dusty particle of a rodlike shape in a twotemperature plasma flow is considered. An analytical model useful for understanding the phenomenon of ion focusing and its effect on the formation of the surface charge of a needle was considered. Pairwise radial correlation functions evaluated in the approximation of binary interaction for moving particles with a stationary Coulomb center. Using conservation law for the momentum ion velocity component has been determined. It was noticed that electron shielding does not influence on the ion focusing density peak value. In that reason ion focusing parameters in the concrete doesn't depend on the electron screening Debye radius. A system with Coulomb particles containing moving point particles, ions and electrons, and fixed spheres have been chosen. The cases of dielectric and metallic dust grains having different orientations with respect to the flow direction are considered.
\end{abstract}

Key words: dusty plasma, ion flow, molecular dynamic, grain charge, drug force.

PACS numbers: 51.50. $+v ; 51.10 .+y ; 52.80 . D y ; 52.25$.Fi.

\section{Introduction}

Dusty plasma is the object of intense experimental and theoretical research of recent years (see, for example, [1-4]). The present work continues a series of studies on numerical simulation of the properties of dusty plasma [5-11]. It investigated processes charging spherical dust particles and the structure of the space charge region around one dust particle placed in a single-temperature, twotemperature, resting $[5,7,8]$ and moving plasma $[6$, $10,11]$, as well as Brownian motion [7] and the dynamics of particulates in the plasma flow $[6,8]$. In $[9,10]$, the mutual influence of the charging of two particles located along the flow and perpendicular to it is considered. In the present work, for the first time, the charge characteristics of a long-form dust particle have been investigated on the basis of a computational experiment, experiments with which are being actively carried out, and the theoretical study of their properties is very limited at the present time (see papers [12-15], in part the results of this paper are given in [11]). In the case of a supersonic ion plasma flow around a negatively charged spherical dust particle (such a situation can occur when a dust particle levitates in the near-electrode plasma layer), a region of increased ionic densityion focus is formed behind the dust.

The process of formation, structure and characteristics of the ion focus are considered in $[5,6]$. In the near-electrode layer, dust particles can form an ordered structure, and ion focusing can affect the interaction of particulates. To describe the interaction of particles in this case, it is not enough to use models based on the introduction of potential forces (the most commonly used is the screened Coulomb potential of Debye-Hückel). An example of this is the effect of ion focusing on the formation of the average charge of a particle in the wake tail considered in $[9,10]$. The performed calculations showed that ion focusing on the first particle can lead to a significant decrease in the negative charge of the second particle due to the increased ion current. In this connection, the question arose about the effect of ion focusing on the charging of particles of an extended shape. In the present work, the formation of a space charge around extended-shape structures in a moving plasma is studied when the ion-focusing effect significantly affects the distribution of the space-charge region around the dust particle [11] 
and accordingly the density of the flux of charged plasma particles varies in different parts of the dust motif of the extended shape.

On the basis of a computational experiment, the charge characteristics of a chain of micron-sized dust particles (macroparticles) that touch each other and are placed in a plasma flow are investigated. Such a chain of dust particles simulates dust particles of extended shape. The cases of a different orientation of the needle relative to the flow are considered, and the dependence of the charge characteristics on the conductivity of the dust particle material is investigated.

\section{Ion focusing in the binary ballistic approx- imation}

Let us first consider an analytical model of ion focusing, which is useful for understanding the phenomenon of ion focusing and its effect on the formation of the surface charge of a needle. We obtain the necessary estimates in the same way as in [5], pairwise radial correlation functions were obtained in the binary interaction approximation of moving particles with a stationary Coulomb center. Let the stationary point Coulomb center (a massive dust particle) with a negative charge $-Z e$ be placed at the origin of coordinates, onto which a uniform plane ion flux with density $N_{0}$, mass $m$, positive charge $e$, velocity $V_{\infty}>0$ flies from infinity. We select the $\mathrm{x}$ axis in the direction of the original ion velocity. Then, in the binary interaction approximation with a point Coulomb center, the ion moves in the collision plane along the trajectory described by the parametric equation [16]:

$$
\begin{gathered}
r=p /(1+\boldsymbol{\varepsilon} \cos \phi), \quad p=\boldsymbol{\rho}_{\infty}^{2} / \boldsymbol{\rho}_{\perp}, \\
\boldsymbol{\varepsilon}=\left(1+\boldsymbol{\rho}_{\infty}^{2} / \boldsymbol{\rho}_{\perp}^{2}\right)^{1 / 2}, \quad \boldsymbol{\rho}_{\perp}=e^{2} Z / m V_{\infty}^{2},
\end{gathered}
$$

where $r$ is the distance to the Coulomb center at some point of the trajectory, $\varphi$ is the angle between the radius vectors at this point and the maximum approach point, $V_{\infty}$ is the velocity at infinity, $\rho_{\infty}$ is an impact parameter, $p$ is the parameter, $\varepsilon$ is the eccentricity of the collision [16 ]. It follows from (1) that an ion incident from infinity in the direction of the $x$ with a velocity $V_{\infty}$ and impact parameter $\rho_{\infty}$, intersects the $x$ axis at some point $x_{0}\left(\rho_{\infty}\right)=\rho_{\infty}^{2} / 2 \rho_{\perp}$. The number of ions that intersect the $x$ axis in unit time in the range $0<x_{0}<x$ $<x_{0}<x_{0}+\Delta x$, is $\Delta j=2 \pi N_{i} V_{\infty} \rho_{\perp} \Delta x$. The flux density per unit length of the $x$ axis does not depend on the point on it.

The component of the ion velocity orthogonal to the $x$ axis at the point of intersection of the $x$ axis is determined by the momentum conservation law and is $V_{\rho}\left(x_{0}\right)=V_{\infty} \rho_{\infty} / x_{0}=V_{\infty}\left(2 \rho_{\perp} / x_{0}\right)^{1 / 2}$. From this it follows that near the axis $\mathrm{x}$ the density of ions is

$$
N(x, \rho)=N_{0}\left(2 \rho_{\perp} x\right)^{1 / 2} / \rho .
$$

This expression, obtained for a collinear flow of non interacting ions, leads to an infinite value of the ion density on the axis. Interaction of plasma particles and thermal velocity spread leads to a blurring of the focusing peak near the $x$ axis. Let us estimate the magnitude of the maximum ion density on the axis with allowance for the smearing due to the thermal velocity of ions $V_{T}=(T / m)^{1 / 2}$.

Let us assume that the thermal velocity of the ions is much less than the velocity of the directed flow. This condition is usually satisfied even for a subsonic flow because of the low ion temperature compared to the electron temperature. Then it is possible to average the density of ions over the radius determined by the thermal velocity of ions $\rho_{0}=x V_{T} / V_{\infty}$.

The number of ions entering a thin cylinder around the $x$ axis in the range $0<x_{0}<x<x_{0}<x_{0}+\Delta$ $x$ for a time $\tau$ is $\Delta N=\tau \Delta x J_{x}$, each of them will be in it for a time $\Delta t=2 \rho_{0} / V_{\rho}$, the cylinder volume is $\Delta V=\pi \rho_{0}^{2} \Delta x$, respectively, the average density in this cylinder $\langle N\rangle=\Delta N \Delta t / \Delta V \tau$. Hence we obtain the following estimate for the ion density near the $x$ axis:

$$
N_{0}(x)=N_{0}\left(8 \rho_{\perp} / x\right)^{1 / 2} V_{\infty} / V_{T},
$$

which in the field of its applicability is in good agreement with the results of calculations of the ion focusing of a plasma flow around one and two macroparticles $[6,8,9]$.

We note that electron shielding practically does not affect the value of the peak of the ion focusing density, so the parameters of ion focusing are practically independent of the electron Debye radius $r_{D e}=\left(T_{e} / 4 \pi N_{e}\right)^{1 / 2}$. A characteristic quantity that determines the parameters of ion focusing is the impact parameter $\rho_{\perp}=e^{2} Z / m V_{\infty}^{2}$, at which the ion 
rotates after a flight near the particle to a right angle. The electronic Debye radius determines not the characteristics of ion focusing, but the scale of the length at which the space charge of the ion focus is screened. Screening by ions under conditions typical for dusty plasma, when $T_{i}<<T_{e}$ and the thermal velocity of ions $V_{T}<<V_{\infty}$, as well as electron shielding, does not play an important role in the formation of the ion focus. However, along with the size of the macroparticle, the Debye ion radius $r_{D i}=\left(T_{i} / 4 \pi N_{i}\right)^{1 / 2}$ is the lower boundary of the region of applicability of formula (3). In the shielding of the charge of a macroparticle by ions, their contribution is approximately the same as that of electrons, since the characteristic kinetic energy of a directed ion flux is of the order of the electron temperature and, accordingly, the effective screening radius of ions roughly coincides with the electronic Debye radius.

\section{Statement of the problem for numerical simulation}

The technique used here to study the properties of a dusty plasma on the basis of the numerical integration of the equations of the dynamics of many particles is described in detail in [5-11]. This technique was adapted to solve the problem of the interaction of a plasma with a dust particle of an extended shape, the cylindrical dust particle was represented as a chain of spheres. If we assume that the charge of each sphere is uniformly along its surface, then the problem reduces to the usual scheme of solutions of the system of equations of motion of molecular dynamics. The errors of this model lead to a loss of information on the distribution of the charge density over a length equal to the radius of the dust particle. Because of this, near the surface of the speck, by reason of the averaging of the charge along its surface, the trajectories of ions and electrons will be inaccurately determined. In addition, some influence is exerted also by the geometric factor of replacement of a body of the cylindrical form, on a chain of spheres. Apparently, to calculate the charging characteristics, we should choose the dimensions of the spheres such that the volume of the cylinder and the total volume of the spheres coincide. But since at this stage of the study we are not talking about an accurate determination of the charging characteristics of a cylinder in plasma, but about the qualitative nature of the charge distribution of long-form dusty grains, the use of such a model seems quite adequate to the purpose of the study.

We consider a system of Coulomb particles consisting of moving point particles, ions and electrons, and fixed spheres. Ions have a mass $M$, a positive charge $e$, electrons have a mass $m$ and a negative charge $-e$. Let us consider the case when all spheres are fixed, have the same radius $\mathrm{R}$, and absorb all the electrons and ions that fall on them. Accordingly, the charge of spheres is determined by the number of absorbed electrons and ions and depends on time. The case of Brownian motion of mobile spheres with variable mass and charge is considered in [7].

We consider the time evolution of a system initially consisting of $2 \boldsymbol{n}$ particles inside a parallelogram $0<x<L x, \quad 0<y<L y, \quad 0<z<L z$. The trajectories of $n$ positive and $n$ negatively charged particles are determined by numerical solution of Newton's equations:

$$
\begin{gathered}
d^{2} r_{k} / d t^{2}=F_{k} / m_{k}, \\
F_{k}=\sum_{l \neq k}^{2 n+n_{g}} f_{k l}, \quad k=1,2, \ldots, 2 n
\end{gathered}
$$

here $r_{k}(t$ is the radius vector of the $\kappa$ - th particle, each of which is characterized by the mass $m_{k}$ and the charge $q_{k}$, and $n_{g}$ is the number of spheres. The Coulomb interaction force $f_{k l}$ between mobile particles at distances between particles less than $r_{0}$ was modernized in accordance with the interaction of uniformly charged mutually permeable spheres of diameter $r_{0}$. This value was chosen much less than the average interparticle distance and such that it did not influence the investigated characteristics. This was usually checked by comparing the results of calculations with various $r_{0}$. When calculating the Coulomb interaction force $f_{k l}$ between moving particles and spheres, it was assumed that the charge of each sphere is uniformly distributed over its surface.

The initial distribution of electrons and ions along the coordinates was chosen to be equiprobable in the volume of the calculated cell. The velocity distribution corresponded to the MaxwellBoltzmann distribution at infinity. Depending on the initial distance to the nearest macroparticle, Maxwell's velocity distribution was shifted by the amount of interaction energy with the macroparticle. The direction of the velocity was chosen isotropic with a constant increment corresponding to the moving plasma. Thus, an initial distribution without bound particles (ions) was formed. In calculating flow characteristics, a sufficiently large initial time 
interval was emitted from consideration, on which the steady-state nature of the flow was established in the gasdynamic sense, and the values of dust charges reached the mean steady-state values.

The number of ions in the system was assumed to be fixed, the number of electrons was variable. The particle-throwing algorithm (instead of the absorbed ones) is used, which accurately preserves the neutrality of the entire system. Instead of the absorbed particle from a randomly chosen point on the surface of the calculated cell, the particles were thrown in such a way as to ensure the neutrality of the entire system, including the charge of the particulates. This procedure consisted in the fact that when the electron was absorbed, their number in the system decreased, and when an ion was absorbed, an electron-ion pair was injected into the system from a random point on the surface. Thus, the number of ions in the system was kept constant, while the number of electrons was variable, ensuring neutrality of the system.

The boundary conditions for modeling the ion flux differed somewhat from those used earlier [6, 8-11], where an absorbing condition was used for the ions at the right boundary and emitting on the left. Here, after the collision of the ion with the wall, it was thrown into the system from a random point on the surface. Moreover, the choice of a point on the surface corresponded to the fact that the distribution function of the particles entering the volume corresponded to the Maxwell distribution shifted by the velocity of the plasma flow. In the absence of absorbing spheres, this procedure yields a uniform plasma flow. When reflecting from the walls for electrons, thermostating boundary conditions were used. Thus, the Maxwell-Boltzmann distribution for electrons and the possibility of appearing in the simulated system of electrons having kinetic energy sufficient to overcome the potential barrier was maintained. This formulation of the problem allows us to take into account the charge and charge fluctuations of a macroparticle in a self-consistent manner.

In addition, a new realization of the boundary conditions makes it possible to simulate the flow of plasma around the dust particle at a lower ion flow rate more correctly than in previous studies, even when the flow velocity is comparable or even much lower than the thermal velocity of the ions. We note that the constant number of ions in the system, if it is possible to reduce the number of electrons due to absorption by a macroparticle, means that the average electron density in the modeled system can be significantly reduced in comparison with the ion density. An estimate of the decrease in electron density is the ratio of the charge of the macroparticle to the number of ions in the system. The situation, when the electron density in the cell is much lower than the ion density, corresponds to the case of dust clouds, crystals, in the near-electrode region the electron density is also lowered.

\section{Results of numerical simulation}

In all the above calculations, we studied a moving two-temperature argon plasma with $z=1$, a temperature of $T_{i}=0.025 \ni B \mathrm{eV}$ and electrons $T_{e}=1 \mathrm{eV}$, the velocity of motion of the plasma corresponded to the kinetic energy of the ions $K_{i}=1{ } \ni B, V_{\infty}=$ $2.2 \times 10^{5} \mathrm{~cm} / \mathrm{s}$, thermal ion velocity $V_{T}=(T / \mathrm{m})^{1 / 2}=$ $2.4 \times 10^{4} \mathrm{~cm} / \mathrm{s}$, ion density $N_{i}=2 \times 10^{12} \mathrm{~cm}^{-3}$. At $N_{i}=$ $2 \times 10^{12} \mathrm{~cm}^{-3}$, the Debye radius (total) is $3.6 \mu \mathrm{m}$, the number of particles in the Debye sphere is 876 . Plasma with such parameters is ideal for both ions and electrons. With the value of the charge of the needle $Q / e=3100$, which is characteristic of the calculations given below, the impact parameter, at which the ion turns at a right angle, is equal to $\rho_{\perp}=e^{2} Z / m V_{\infty}^{2}=2.2 \times 10^{-4} \mathrm{~cm}$.

The calculated region was a parallelogram of size $20 \mu m \times 10 \mu m \times 10 \mu m$, in which 4000 ions were located. There were 6 spheres in the simulated volume, absorbing electrons and ions, touching each other and located along a straight line lying in the $x y$ plane at $z=5 \mu \mathrm{m}$.

Fig. 1 illustrates three variants of the arrangement of spherical dust particles that touch each other and imitate a needle. The angle $\alpha$ characterizes the inclination of the needle relative to the ion flow. Variant a) corresponds to the location of the needle along the stream, the angle $\alpha=0$, the coordinates of the sphere centers $x=5,6,7,8,9,10 \mu m, y=z=5$ $\mu \mathrm{m}$, with a radius $R=0.5 \mu \mathrm{m}$. Variant b) corresponds to the location of the needle at an angle $\alpha=$ $\pi / 4$ to the direction of the ion flow, variant c) corresponds to the location of the axis of the needle perpendicular to the flow. The calculation time for these results was $26 \mathrm{~ns}$, the ion Langmuir period was $21 \mathrm{~ns}$. When calculating the average charging characteristics of the dust chain in the table and in the figures, the initial time relaxation region was ejected, which usually amounted to several ion Langmuir periods. 
Table 1 - Integral charging characteristics of the entire chain of dust particles (calculations №№1-6), depending on the conductivity of the surface $\sigma s$ and the angle of inclination of the needle to the flux $\alpha$. Calculation №7 is the characteristics of a single spherical speck of dust in the same flow. The time averages are given: the charge in electron charge units, the ion flux normalized to the unperturbed stream $J_{0}=\pi R^{2} N_{i}\left(2 K_{i} / M\right)^{1 / 2}$, the average potential over surface $\varphi_{s}$, normalized to the electron temperature, the projections on the $x, y$ axis of the dipole moment of the entire Coulomb system normalized by the quantity $P_{0}=e N_{i}^{-1 / 3}$.

\begin{tabular}{|c|c|c|c|c|c|c|c|}
\hline № & $\boldsymbol{\sigma}_{\mathbf{s}}$ & Angle & $-\mathbf{Q} / \mathbf{e}$ & $\mathbf{J} / \mathbf{J}_{\mathbf{0}}$ & $-\mathbf{e} \boldsymbol{\varphi}_{\mathbf{s}} / \mathbf{T}_{\mathbf{e}}$ & $\left\langle\mathbf{P}_{\mathbf{x}} / \mathbf{P}_{\mathbf{0}}\right\rangle$ & $\left\langle\mathbf{P}_{\mathbf{y}} / \mathbf{P}_{\mathbf{0}}>\right.$ \\
\hline 1 & 0 & 0 & 3157 & 125 & 3.08 & 9084 & -118 \\
\hline 2 & 0 & $\pi / 4$ & 3242 & 251 & 2.80 & 11090 & 1485 \\
\hline 3 & 0 & $\pi / 2$ & 3199 & 187 & 2.80 & 11580 & 330 \\
\hline 4 & $\infty$ & 0 & 3087 & 51 & 2.61 & 7301 & 3 \\
\hline 5 & $\infty$ & $\pi / 4$ & 3159 & 246 & 2.74 & 10410 & 1165 \\
\hline 6 & $\infty$ & $\pi / 2$ & 3220 & 192 & 2.83 & 11980 & 255 \\
\hline 7 & \multicolumn{2}{|c|}{ one particle } & 1214 & 9 & 3.02 & 239 & -10 \\
\hline
\end{tabular}

In the table, the integral characteristics of the needles for different types of surface and the angle of inclination with respect to the flow are given, in addition, for comparison, the charging characteristics of one dust particle of the same size under similar conditions are included. In addition, the dipole moment of the entire system is calculated. Since the total charge of the system is zero, the magnitude of the dipole moment does not depend on the choice of the coordinate system - its projection to the $x$ axis is equal to $P_{x}=\sum x q$, the summation is over all particles. The projection of the dipole moment onto the $y$ axis is calculated in a similar way. a)

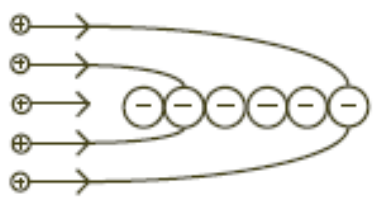

b)

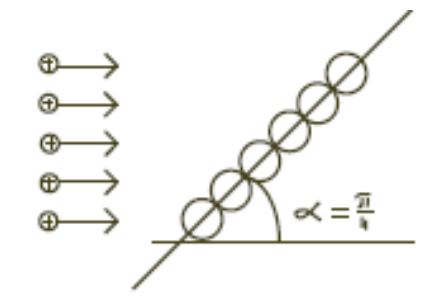

c)

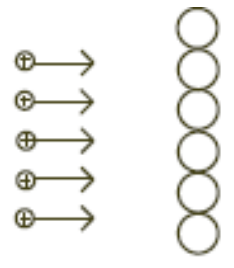

Figure 1 - Scheme of arrangement of a chain of dust particles (needle) relative to the ion flow:

a) along the stream, the angle $\alpha=0$, the coordinates of the sphere centers $x=5,6,7,8,9,10 \mu m, y=z=5 \mu m$, with a radius $R=0.5 \mu \mathrm{m} ; \mathrm{b}$ ) at an angle $\alpha=\pi / 4$ to the direction of the ion flow; c) perpendicular to the flow

In Figure 2 shows the charging characteristics of dielectric dusty grains that do not conduct a charge and are located along the ion flow. Histograms are given that characterize: a) the value of the average negative charge of the speck of dust; b) the flux of ions per dust particle, normalized by a value $J_{0}=\pi R^{2} N_{i}\left(2 K_{i} / M\right)^{1 / 2}$ that is equal to the gasdynamic flow of cold ions absorbed by the sphere, moving in the same direction and having directional energy $K_{i} ;$ c) the average surface potential of the dust particles, determined by all the plasma particles and the surface charge of all the dust particles. When calculating the surface potential of a dusty grain, it was assumed that the charge along the surface of the dust particle was distributed uniformly.
In this case, the average potential over the surface from the sphere averaged over the surface of the other sphere is equal to the charge potential at the center of these spheres. In this case, a known property of the electrostatic potential is used, that its average value over the sphere is equal to its value at the center of the sphere (for a charge located outside the sphere). It is obvious that the error with this calculation of the average potential is very insignificant.

The results corresponding to this calculation are given in the first line of the table - calculation №1. The time-average charge of the entire needle, the ion flux absorbed by it, and the surface potential are presented. As the surface potential for the dielectric 
chain of dust particles, an average of 6 dusty grains is given. The table also presents the projections of the dipole moment of the entire system. Charging characteristics of dusts that do not conduct an electric charge and are located along the flow (calculation №1 from the table): a) the value of the average negative charge of the dust particle; b) the flux of ions per dust particle, normalized by the flow $\left.J_{0}=\pi R^{2} N_{i}\left(2 K_{i} / M\right)^{1 / 2} ; \mathrm{c}\right)$ average surface potential of dust particles.

a)

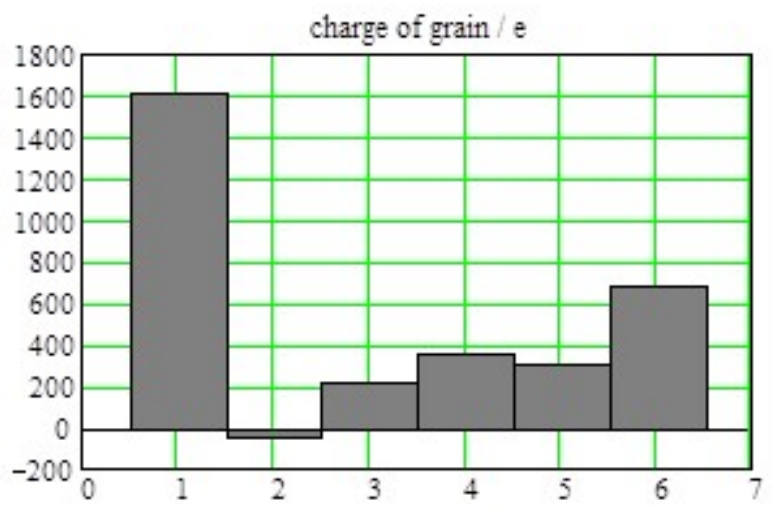

b)

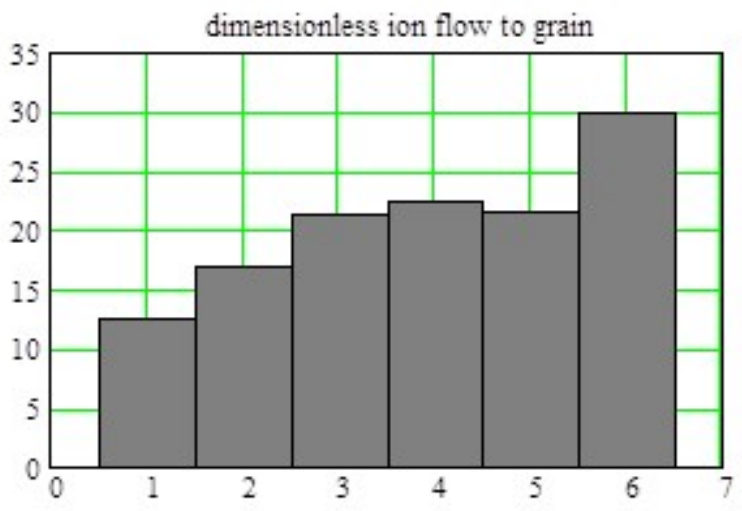

c)

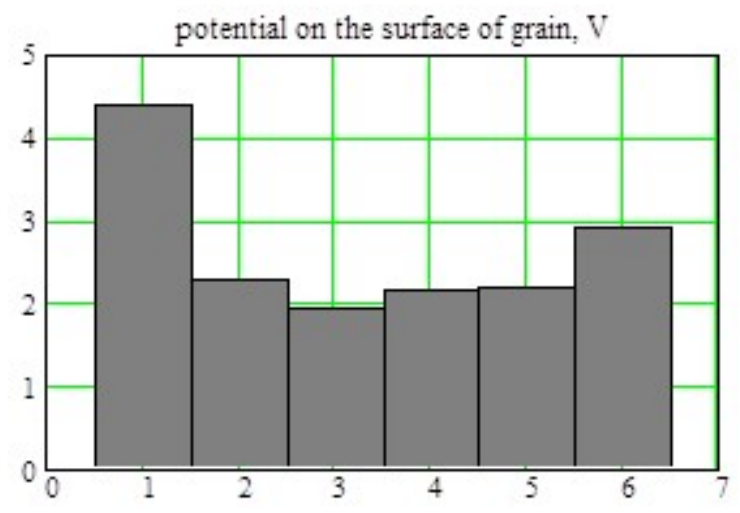

Figure 2 - Charging characteristics of dusts that do not conduct an electric charge and are located along the flow (calculation №1 from the table):

a) the value of the average negative charge of the dust particle;

b) the flux of ions per dust particle, normalized by the flow $J_{0}=\pi R^{2} N_{i}\left(2 K_{i} / M\right)^{1 / 2}$;

c) average surface potential of dust particles 
In Figure 3 and 4 show the results of calculating the densities of ions and electrons around dielectric dust particles. In Figure 3 shows the ion densities in the $x y$ plane passing through a chain of dust particles. Here and below, in all the figures, the values of the density are normalized to the mean ion density $N_{i}=$ $2 \times 10^{12} \mathrm{~cm}^{-3}$. In Figure 3a shows the distributions of the ion density along the $x$ axis at $y=0.1,1,2,3,4,5$ $\mu m$. The maximum ion density, which exceeds the average density by about 8 times, is reached immediately behind the dusty grain. The estimate from formula (3) gives for this point a density exceeding by about 16 times. Obviously, the difference is due to the influence of the fineness of the size of the dust particles (the shadow effect), the screening of the charge of the needle and the repulsion of the ions near the peak density. Obviously, all these factors lead to dissipation - the spreading of the peak density. In general, estimates (2) and (3) correctly describe the behavior of the density in the ion focus (wake tail). In Figure $3 b$ shows lines of equal level in the $x y$ plane passing through a chain of dust particles. In Fig. 3c shows a three-dimensional image of the ion density surface in the $x y$ plane passing through a chain of dust particles. In Figure 4 shows similar distributions for the electron density.

a)

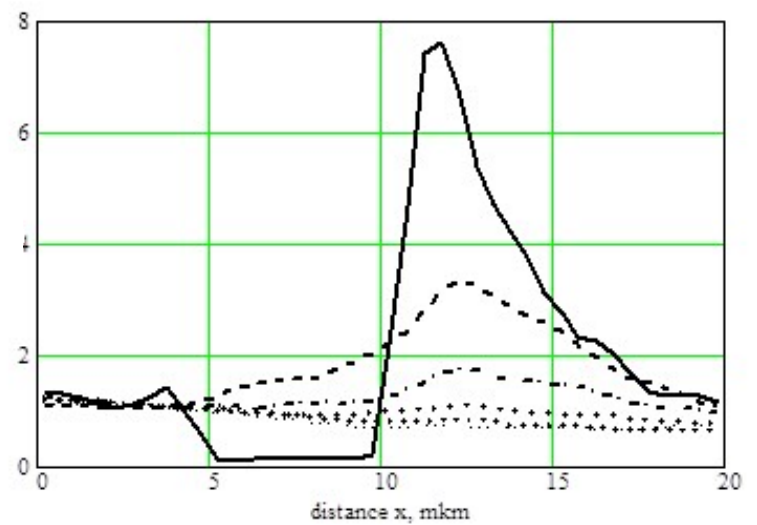

b)

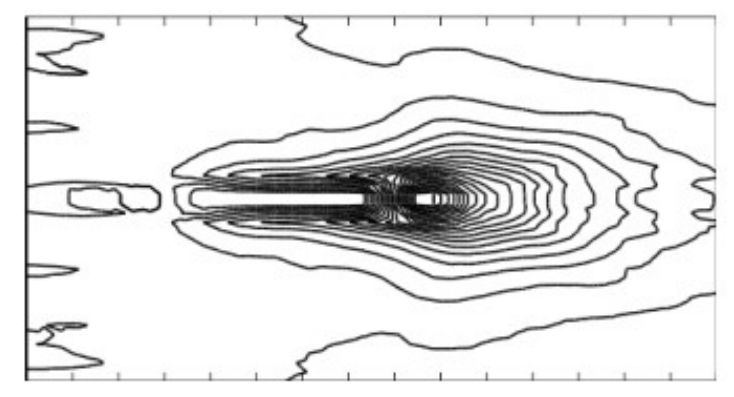

c)

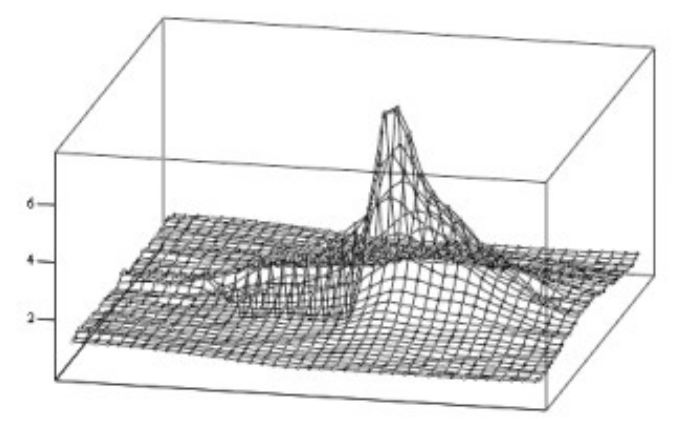

Ions

Figure 3 - Density of ions in the $x y$ plane passing through a chain of dielectric dusts:

a) the ion density distribution along the $x$ axis at $y=0.1,1,2,3,4,5 \mu \mathrm{m}$;

b) a line of equal level in the $x y$ plane passing through a chain of dust particles;

c) three-dimensional image of the ion density surface in the $x y$ plane passing through a chain of dust particles 
a)

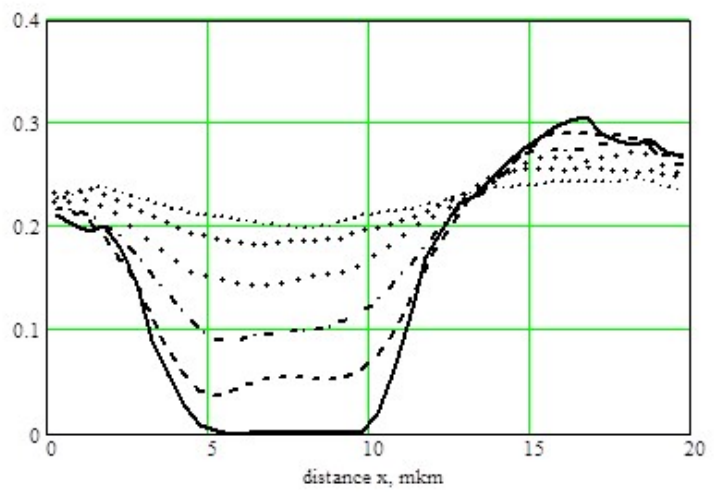

b)

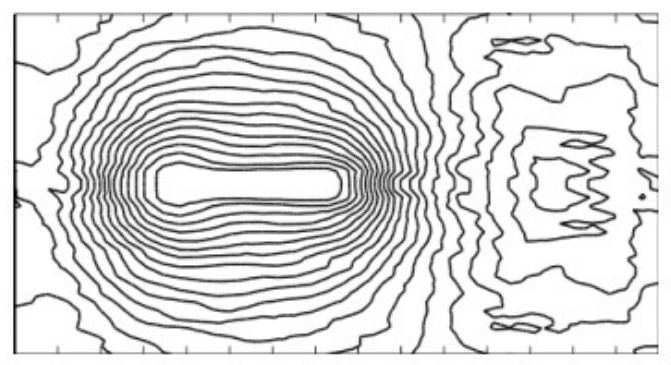

Electrons

c)

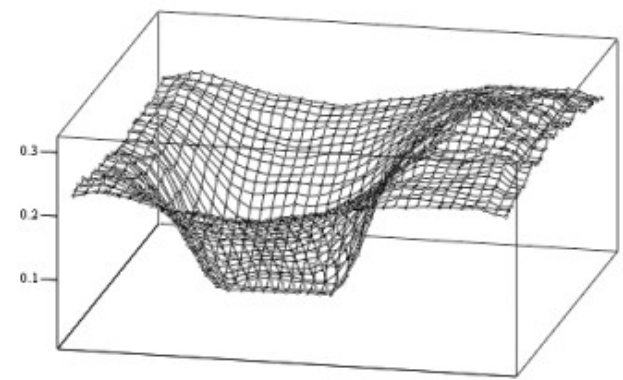

Electrons

Figure 4 - Similar Figure 3 distribution for the electron density

In Figure 5 shows the characteristics for a charge-conducting needle oriented along the ion flow - calculation No. 4 in the table. Here are the histograms characterizing: a) the value of the average negative charge of a dusty grain; b) the flow of ions to the dust particle; c) the flow of electrons to the dust particle. The fluxes of ions and electrons to the dust particle, as in Figure 2 are normalized by magnitude $J_{0}$. In contrast to the calculation of dielectric dust grains, the surface potential of metallic dusts does not change, because dust particles touch each other, but the fluxes of ions and electrons vary significantly. In Figure 5 a) circles with a thick dot in the center indicate the values of the equilibrium charge obtained from the simplest model of the charge distribution on metallic spheres placed not in the plasma but in vacuum. In this model, it was assumed that the total charge $Q=3087 e$ obtained in the calculation of №4 was redistributed over the spheres in such a way that the surface potentials of all the dust particles were equal. When calculating the surface potential of a dust particle, it was assumed that the charge along the surface of the dust particle is uniformly distributed, respectively, the average potential from other spheres is equal to the potential determined through the distances between their centers. In Figure 6 shows the charging characteristics of the needles located at angles $\alpha=\pi / 4$ and $\alpha=\pi$ / 2 to the flow - calculations №2, 3, 5, 6 from the table. In Figure 6a) the values of the mean negative charge of dust particles are given; in Figure 6b) is the average surface potential of the dust particles. 
a)

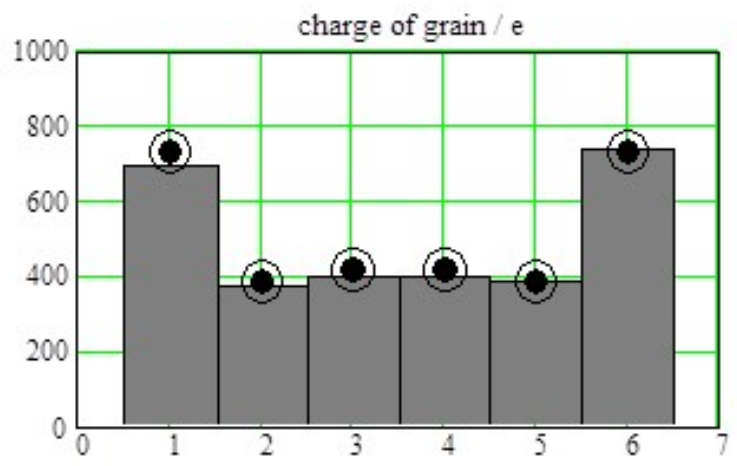

b)

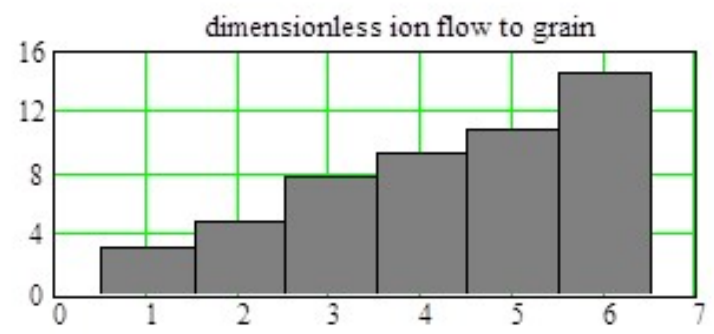

c)

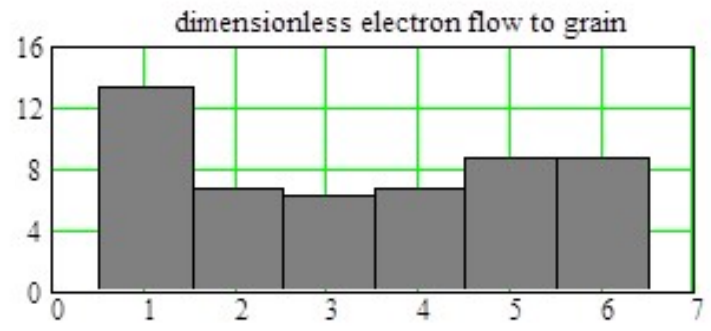

Figure 5 - Charging characteristics of dust particles conducting electric charge and located along the flow (calculation №4 from the table):

a) the value of the average negative charge of the dust particle;

b) the flow of ions,

c) the flow of electrons to the dust particle, normalized to the flow

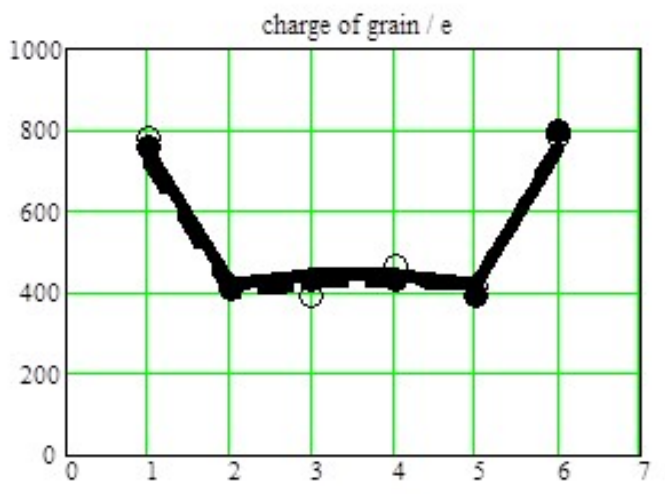

a)

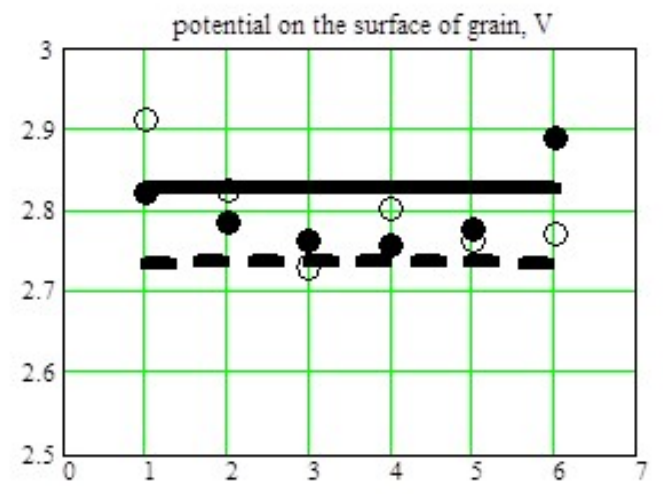

b)

Figure 6 - Characteristics of charge of dust particles when the needle is placed at angles $\alpha=\pi / 4$ and $\alpha=\pi / 2$ to the flow - calculations №2, 3, 5, 6 from the table (circles, dots, dashed curves and solid curves, respectively): a) the value of the average negative charge of dust particles;

b) the average surface potential of the dust particles 


\section{Discussion}

The results of the calculations show two interesting new effects: the effect of ion focus on the character of charge of a long needle and a strong dependence on the conductivity of the surface of the dust particles. Strictly speaking, to ensure the equipotentiality of the surface, there is no need for the entire dust particle (its volume) to be conductive (metallic). The finite surface conductivity (even rather low) may be sufficient to ensure equipotentiality. Since the surface of the dust particles is exposed to particles of plasma and radiation, surface conductivity can also occur in dielectric materials. This possibility must be taken into account in interpreting the experiments.

Let us first consider the charging characteristics of the needles oriented along the ion flow. In this case, the total charge of dust particles for both types of surface differs insignificantly (for an insulator it is approximately $2 \%$ higher), but the nature of the distribution of charge density along a dusty grain varies radically. In a dielectric dusty grain, about half of the charge is concentrated at its beginning, and then there is an area in which the charge density even changes sign. This effect is due to focusing into this part of the dust particle a more significant ion flux and a corresponding decrease in the electron flux due to the geometric factor. As a result, the dielectric dusty grain has a significant dipole moment, which can have a significant effect on its stability and lead to the appearance of oscillations [13] and rotation [14].

Another interesting feature is that the flow of plasma particles to a dielectric dusty grain is approximately two and a half times higher than that of a metallic particle (with an approximately equal total charge - see table, calculations №1, 3). That is, the dielectric speck because of the accumulation of charge in its nose more strongly focuses the ion stream on its tail. Accordingly, a large drag force on the ion side, due to the momentum transfer during collision, acts on the dielectric dust particle.

Let us now consider the charging characteristics of the needles oriented at an angle to the direction of the ion flux. In this case, the total charge of the needles also differs insignificantly and practically does not depend on either the angle or the type of surface (see table, calculations №2, 3, 5, 6). The nature of the distribution of the charge density along the needle is the same for all calculations, see Fig. 6 a) and is close to the distribution of the charge density distribution of a conducting dust, located along the flow. Accordingly, for all these calculations, the distribution of the surface potential of the needles is also close.

An interesting feature is the appearance of a significant dipole moment in a direction perpendicular to the flow, with an acute "angle of attack" $\alpha=\pi / 4$. This effect, together with the dependence of the drag force on the angle, opens up rich possibilities for the appearance of rotational oscillations, a simple analytical model of which was considered in [14]. We note that an attempt has also been made in [15] to study theoretically the behavior of charged needles in an ion beam. But since it used the model of a very long needle and did not take into account the focusing of ions, the comparison of the results obtained there with the present calculations goes beyond the range of applicability of the theoretical model.

\section{Conclusions}

The results of numerical simulation lead to the need to rethink many phenomena observed in the near-electrode layer containing dust particles of extended shape. The inhomogeneity of their charging, the large dipole moment of the needle itself and the surrounding plasma (see the experimental results in [15]) lead to a very complex interaction of the needles between themselves and the surrounding plasma. Although the developed model is the first numerical experiment to investigate the electrical characteristics of spindle-shaped structures located in the ion flow of the near-electrode layer and, in spite of sufficiently significant assumptions embedded in the numerical model, the calculations make it possible to clarify the character of the charge formation of long-sized dust grains, to analyze the kinetic processes leading to formation of dust structures in the plasma, to test theoretical models [17 - 22]. These results are important for predicting processes under experimental conditions.

\section{Acknowledgements}

This work was supported in part by the Russian Science Foundation, project no. 14-50-00124. The numerical simulations were carried out at the Joint Institute for High Temperatures, Russian Academy of Sciences. 


\section{References}

1. V.N. Tsytovich. Dust plasma crystals, drops, and clouds // Phys. Usp. - 1997. - Vol. 40. - P. 53.

2. V.E. Fortov, A.G. Khrapak, A.V. Ivlev, S. A. Khrapak, G.E. Morfill. Complex (dusty) plasma: curent status, open issues, perspectives // Physics Reports. - 2005. - Vol. 421. - No. 1-2. - P. 1.

3. S.V. Vladimirov, K. Ostrikov, A.A. Samarian. Physics and applications of complex plasmas. - London: Imperial College, 2005. - 439 p.

4. G.E. Morfill, A.V. Ivlev. Complex plasmas: An interdisciplinary research field // Rev. Mod. Phys. - 2009. Vol. 81. - P. 1353.

5. S. A. Maiorov. Pair correlation function for a dusty plasma // Plasma Phys. Rep. - 2000. - Vol. 26. - P. 628.

6. S. A. Maiorov, S. V. Vladimirov, N. F. Cramer. Plasma kinetics around a dust grain in an ion flow // Phys. Rev. E. - 2001. - Vol. 63. - P. 017401.

7. A.M. Ignatov, S.A. Trigger, S.A. Maiorov, W. Ebeling. Rotational kinetics of absorbing dust grains in neutral gas // Phys. Rev. E. - 2002. - Vol. 65. - P. 046413.

8. S. A. Maiorov, S.V. Vladimirov, N.F. Cramer. Calculation of the grain charge fluctuation in a dusty plasma// Plasma Phys. Rep. - 2002. - Vol. 28. - P. 946.

9. S.V. Vladimirov, S.A. Maiorov, N.F. Cramer. Kinetics of plasma flowing around two stationary dust grain // Phys. Rev. E. - 2003. - Vol. 67. - P. 016407.

10. S.V. Vladimirov, S.A. Maiorov, O. Ishihara. Molecular dynamic simulation of plasma flow around two stationary dust grains // Phys. of Plasm. - 2003. - Vol. 10. - P. 3867

11. S. A. Maiorov. Charging of rod like grain in plasma flow // Plasma Phys. Rep. - 2004. - Vol. 30. - P. 766.

12. B. M. Annaratone, A. G. Khrapak, A.V. Ivlev, G. Solner, P. Bryant, R. Sutterlin, U. Konopka, K. Yoshino, M. Zuzic, H.M. Thomas, G.E. Morfill. Levitation of cylindrical particles in the sheath of an RF plasma // Phys. Rev. E. 2001. - Vol. 63. - P. 036406.

13. S. V. Vladimirov, E.N. Tsoy. Oscillation in a chain of rod-shaped colloidal particles in a plasma // Phys. Rev. E. - 2001. - Vol. 64. - P. 035402.

14. M. P. Hertzberg, S.V. Vladimirov, N. F. Cramer. Rotational modes of oscillation of rod like dust grains in plasma // Phys. Rev. E. - 2003. - Vol. 68. - P. 026402.

15. A.V. Ivlev, A.G. Khrapak, S.A. Khrapak, B.N. Annaratone, G. Morfill, K. Yoshino. Rod like particles in gas discharged plasmas: Theoretical model // Phys. Rev. E. - 2003. - Vol. 68. - P. 026404.

16. L. D. Landau, E. M. Lifshitz, Mechanics - New York: Pergamon Press, 1988. - 169 p.

17. A. A. Samarian, S. V. Vladimirov. Rotational dust particles alignments in a plasma sheath // Contrib. Plasma Phys. - 2009. - Vol. 49. - P. 260-280.

18. S.A. Maiorov, B.A. Klumov. On the Interaction of microparticles with ion flux in gas discharge Plasma// Bulletin of the Lebedev Physics Institute. - 2013. - Vol. 40. - P. 285.

19. L. R. Marcus, B. M. Annaratone, H. M. Thomas, G. E. R. Morfill. Experiments with microrods in a radiofrequency plasma sheath // Phys. Plasm. - 2006. - Vol. 13. - P. 063502.

20. A. A. Samarian, S. V. Vladimirov. Rotational dust particles alignments in a plasma sheath // Contrib. Plasma Phys. - 2009. - Vol. 49. - P. 260-280.

21. O.S. Vaulina, I.I. Lisina, K.G. Koss. Formation of chain structures of cylindrical dust particles in a weacly ionized plasma // Journal of Experimental and Theoretical Physics. - 2014. - Vol. 119. - P. 772.

22. I. Lisina, E. Lisin, O. Vaulina. Spatial configurations of charged rod-like particles in external electric field // Physics of Plasmas. - 2016. - Vol. 23. - P. 033704. 